\title{
Synchronous Onset of Two Complications in Colon Cancer: A Case Report
}

\author{
Andrej Nikolovski ${ }^{\mathrm{a}, \mathrm{b}}$, Nimetula Limani ${ }^{\mathrm{a}}$, Ana Lazarova ${ }^{\mathrm{a}}$, Shenol Tahir ${ }^{\mathrm{a}}$
}

\begin{abstract}
As one of the most frequent malignancies in the world's population, colorectal cancer is also associated with its complications (perforation, obstruction and bleeding). Their incidence is common (2.6-50\%), but synchronous occurrence of two of them in a patient is a rare condition and it is reported in small series or as a case report in the literature. We present a case of the descending colon cancer in a female patient that presented with perforation on tumor site with consecutive retroperitoneal and lumbar abscess and simultaneous presentation of large bowel obstruction. The patient was admitted and operated the same day, the abscess was opened and drained and bowel resection was performed with colostomy creation. The postoperative recovery was uneventful and the patient was discharged on postoperative day 10. Due to the high morbidity/mortality in cases of colon cancer emergencies, Hartman's procedure is often a reasonable option. Timely diagnosis of the colon cancer can reduce the complication rate of the disease worldwide.
\end{abstract}

Keywords: Colon cancer complications; Obstruction; Perforation

\section{Introduction}

As the third most incident malignancy in the world population, colorectal cancer (CRC) is responsible for $11 \%$ of all cancer diagnoses per year. Southern Europe, Australia with New Zealand and Northern Europe are the regions of highest incidence if only the colon cancer is considered [1]. With this high incidence among the world population, certain types of complications directly related to colon cancer that require some type of emergency intervention can be expected.

Typical colon cancer complications are bleeding, obstruction and perforation. Up to $33 \%$ of the patients with CRC present with some type of these emergencies [2].

Most common is the colon obstruction due to the local

Manuscript submitted February 20, 2021, accepted March 8, 2021

Published online March 24, 2021

aUniversity Surgical Clinic "St. Naum Ohridski”, Skopje, North Macedonia bCorresponding Author: Andrej Nikolovski, University Surgical Clinic "St. Naum Ohridski", Skopje, North Macedonia.

Email: andrejnikolovski@ymail.com

doi: https://doi.org/10.14740/jmc3684 tumor growth with incidence ranging between $15 \%$ and $29 \%$ [3]. Large bowel obstruction is also the most common cause for emergency surgical colon procedures [4].

Second most common complication of CRC is the perforation with overall incidence of $2.6 \%$ up to $12 \%$ [5]. Colon perforation can occur on tumor site or distantly (proximal to the obstructing tumor). Perforation on tumor site can result in free perforation or a contained one with an abscess formation and is caused by tumor necrosis and tissue friability. Distant perforation occurs due to the increasing intraluminal pressure and consequent wall necrosis and perforation (law of Laplace) in the most wide parts of the colon (cecum, ascending and transverse colon) [6].

Even though bleeding from CRC is reported in up to $50 \%$ of cases [2], it is usually occult and self-limited. Acute massive bleeding from CRC is rare [6].

Synchronous occurrence of more than one of the above complications of CRC is rare. Kyllonen reports an overall incidence of tumor perforation with or without obstruction in only $4 \%$ of patients [7]. Glenn and McSherry report a dual complication incidence (obstruction and perforation) of $1.7 \%$. Almost in all of their patients, the perforation and obstruction occurred at tumor site. Only one patient with transverse colon cancer had distant (cecal) perforation [8]. In a retrospective study of 1,850 patients with CRC, Chen and Sheen-Chen found 2.6\% of patients had obstruction with perforation [9].

\section{Case Report}

\section{Investigations}

We present a case of a 61-year-old female patient that presented to our emergency department. On admission, she was barely standing on her feet and she complained of general weakness, strong pain in the left lumbar region, high fever, absence of flatus and stool passage.

Physical examination revealed clinical signs of bowel obstruction. In the left lumbar region, there was a subcutaneous palpable and painful tumor with local skin inflammation.

\section{Diagnosis}

Laboratory test showed hemoglobin value of $111 \mathrm{~g} / \mathrm{L}$ (120.0 $165.0 \mathrm{~g} / \mathrm{L})$, leukocyte count of $17.9 \times 10^{9} / \mathrm{L}\left(3.8-5.8 \times 10^{9} / \mathrm{L}\right)$, serum $\mathrm{Na}^{+}$of $132.8 \mathrm{mmol} / \mathrm{L}(137.0-147.0 \mathrm{mmol} / \mathrm{L})$, serum al- 


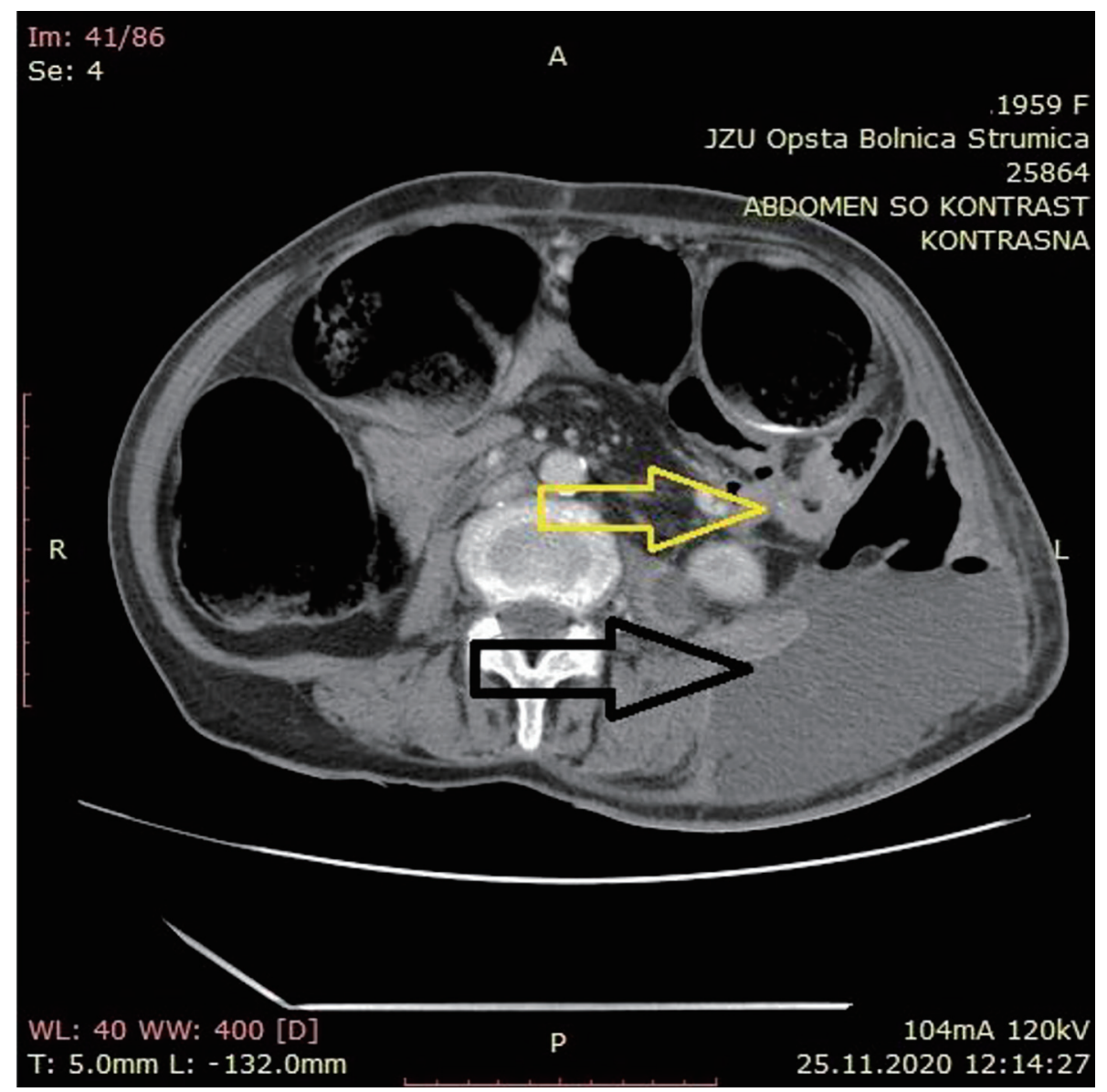

Figure 1. Contrast-enhanced abdominal computed tomography scan with suspicious neo-infiltrative obstructing lesion (yellow arrow) and retroperitoneal abscess that spreads in the left lumbar region with a bubble-gas level (black arrow).

bumin level of $28.7 \mathrm{~g} / \mathrm{L}(35.0-52.0 \mathrm{~g} / \mathrm{L})$ and C-reactive protein level of $262.4 \mathrm{mg} / \mathrm{L}(0-5.0 \mathrm{mg} / \mathrm{L})$. Other laboratory parameters were within normal limits. Contrast-enhanced computed tomography (CT) scan was previously done in other medical institution. It revealed large bowel obstruction with a site of obstruction at the level of the descending colon where a suspicious neo-infiltrative obstructing lesion was detected (yellow arrow). The scan also showed a retroperitoneal abscess that spreads over the left lumbar region with a bubble-gas level (black arrow) (Fig. 1).

\section{Treatment}

After admission and short resuscitation, the patient was referred to the operating room for an exploratory laparotomy. During exploration, a distended colon was found due to obstructing neo-infiltrative and perforated process of the descending colon. After opening the retroperitoneum, pus was freed from the abscess cavity. Its microbiological analysis showed presence of coliform bacteria. A proper lavage and drainage of the abscess cavity was done. Resection of the descendent colon was followed, with a creation of a terminal transversostomy at the left side of the abdominal wall.

\section{Follow-up and outcome}

The pathology report showed a moderately differentiated obstructive adenocarcinoma of the descending colon with a $1 \mathrm{~cm}$ wide defect on the colon wall. The process invaded the serosa of the colon with pericolic infiltration of the fatty tissue. Only five lymph nodes were yielded and analyzed (one of them had metastatic deposit). The final stage according to the eighth edition of Union for International Cancer Control (UICC) was pT4a pN1c pM0, pL1, pV0, pR0, G2, NG2, stage III B.

The postoperative period of recovery was slow but uneventful. Liquid oral intake started at postoperative day 3 and solid food was administered after the passage of gas through the formed stoma (day 4). The patient was discharged on postoperative day 10 . There was no wound infection or any other accompanied postoperative morbidity.

\section{Discussion}

Treatment strategies in the onset of emergency colon cancer are defined. According to the 2017 World Society of Emergency Surgery (WSES) guidelines on colon and rectal cancer 
emergencies, in cases of transverse/left colon cancer perforation, resection with primary anastomosis should be attempted. Hartmann's resection is an option, also. As bottom line, the surgeon should decide whether to create stoma or choose a one-stage surgery with primary anastomosis [10].

Fact speaking in favor of diverting stoma creation after the resection is the high mortality rate after single-stage surgery in perforated and/or obstructed colon cancer [11].

Another unfavorable moment for primary anastomosis creation in perforated left colon cancer is the anastomotic leak. The rates range from $3.5 \%$ to $30 \%$ [12].

When choosing a type of surgery for left colon cancer obstruction, main comparison is made between single-stage surgery and Hartmann's procedure. Lately, no randomized controlled trials were conducted. Therefore, there is no evidence available and the final choice for the type of the procedure is the surgeon's one [10].

In our case report, we considered Hartmann's procedure as the most reasonable and appropriate choice.

\section{Conclusion}

Due to the high incidence of colon cancer, its complications are still frequent too. They can be a real obstacle in performing one seriously planned single-stage oncologic surgical procedure with the least possible postoperative adverse events. Therefore, timely diagnosis can result in decreased number of complicated colon cancer cases.

\section{Acknowledgments}

None to declare.

\section{Financial Disclosure}

None to declare.

\section{Conflict of Interest}

None to declare.

\section{Informed Consent}

Not applicable because the manuscript and the images show nothing from the patient's identity.

\section{Author Contributions}

A. Nikolovski and N. Limani were involved in the operation and the postoperative treatment of the patient. A. Nikolovski made the conception and the design of article and drafted the manuscript. A. Lazarova interpreted the CT finding from the other medical institution. All authors read and critically reviewed the report. All authors approved the final submitted version.

\section{Data Availability}

The authors declare that data supporting the findings of this study are available within the article.

\section{References}

1. Bray F, Ferlay J, Soerjomataram I, Siegel RL, Torre LA, Jemal A. Global cancer statistics 2018: GLOBOCAN estimates of incidence and mortality worldwide for 36 cancers in 185 countries. CA Cancer J Clin. 2018;68(6):394424.

2. Barnett A, Cedar A, Siddiqui F, Herzig D, Fowlkes E, Thomas CR, Jr. Colorectal cancer emergencies. J Gastrointest Cancer. 2013;44(2):132-142.

3. Ohman U. Prognosis in patients with obstructing colorectal carcinoma. Am J Surg. 1982;143(6):742-747.

4. Teixeira F, Akaishi EH, Ushinohama AZ, Dutra TC, Netto $\mathrm{SD}$, Utiyama EM, Bernini CO, et al. Can we respect the principles of oncologic resection in an emergency surgery to treat colon cancer? World J Emerg Surg. 2015;10:5.

5. Larsson B, Perbeck L. The possible advantage of keeping the uterine and intestinal serosa irrigated with saline to prevent intraabdominal adhesions in operations for fertility. An experimental study in rats. Acta Chir Scand Suppl. 1986;530:15-18.

6. Baer C, Menon R, Bastawrous S, Bastawrous A. Emergency Presentations of Colorectal Cancer. Surg Clin North Am. 2017;97(3):529-545.

7. Kyllonen LE. Obstruction and perforation complicating colorectal carcinoma. An epidemiologic and clinical study with special reference to incidence and survival. Acta Chir Scand. 1987;153(10):607-614.

8. Glenn F, McSherry CK. Obstruction and perforation in colo-rectal cancer. Ann Surg. 1971;173(6):983-992.

9. Chen HS, Sheen-Chen SM. Obstruction and perforation in colorectal adenocarcinoma: an analysis of prognosis and current trends. Surgery. 2000;127(4):370-376.

10. Pisano M, Zorcolo L, Merli C, Cimbanassi S, Poiasina E, Ceresoli M, Agresta F, et al. 2017 WSES guidelines on colon and rectal cancer emergencies: obstruction and perforation. World J Emerg Surg. 2018;13:36.

11. Gullino D, Giordano O, Masella M, Lijoi C, De Carlo A. [The single-stage surgery of perforated colon carcinoma. Our experience of 46 cases]. Minerva Chir. 1999;54(3):127-137.

12. Veyrie N, Ata T, Muscari F, Couchard AC, Msika S, Hay JM, Fingerhut A, et al. Anastomotic leakage after elective right versus left colectomy for cancer: prevalence and independent risk factors. J Am Coll Surg. 2007;205(6):785793. 